\title{
Lack of Knowledge About Surgical Smoke, Masks, and Respirators Among US Dermatology Residents and Fellows in the Era of COVID-19
}

Maggie Chow, MD, PhD; Shang I. Brian Jiang, MD

\section{PRACTICE POINTS}

- Harmful surgical smoke is created with electrocautery. Smoke evacuators and approved surgical masks can help mitigate the harmful effects of smoke on the health of dermatologic surgeons.

- Dermatology curricula for residents and medical students should include information on surgical smoke safety. for dermatologic surgeons, and it is not yet known if this population is at higher risk for COVID-19 infection due to aerosolized viral particles in the air or in surgical smoke. Nonetheless, we feel that additional safety measures during dermatologic surgery are warranted, particularly when operating on areas of higher viral load such as the nasal or oral mucosae, and understanding of safety equipment is paramount. Thus, we aimed to assess the awareness of safety measures among training dermatologists and dermatologic surgeons during the COVID-19 pandemic.

In April 2020, one of the authors (S.I.B.J.) gave a lecture to residents and fellows of accredited dermatology residency or fellowship programs in the United States on surgical masks and surgical smoke in dermatologic surgery on an online videoconferencing platform through our institution. During the lecture, participants were polled regarding their understanding of these topics. Forty-one attendees were included in this analysis, with a $100 \%$ response rate. Results showed that 54\% (22/41) of respondents indicated they had not had formal lectures on surgical smoke content and management, and 51\% (21/41) responded that they do not use a smoke evacuator during surgical procedures. When asked why smoke evacuators are not used, $27 \%(11 / 41)$ responded that the equipment is too cumbersome, $12 \%$ (5/41) reported that smoke evacuators are not available at their practice or institution, $7 \%$ did not believe that smoke evacuators are

CONTINUED ON PAGE 212

From the Department of Dermatology, University of California, San Diego.

The authors report no conflict of interest.

Correspondence: Shang I. Brian Jiang, MD, Department of Dermatology, University of California, San Diego, 8899 University Center Ln, Ste 350,

San Diego, CA 92122 (bjiang@ucsd.edu).

doi:10.12788/cutis.0091 


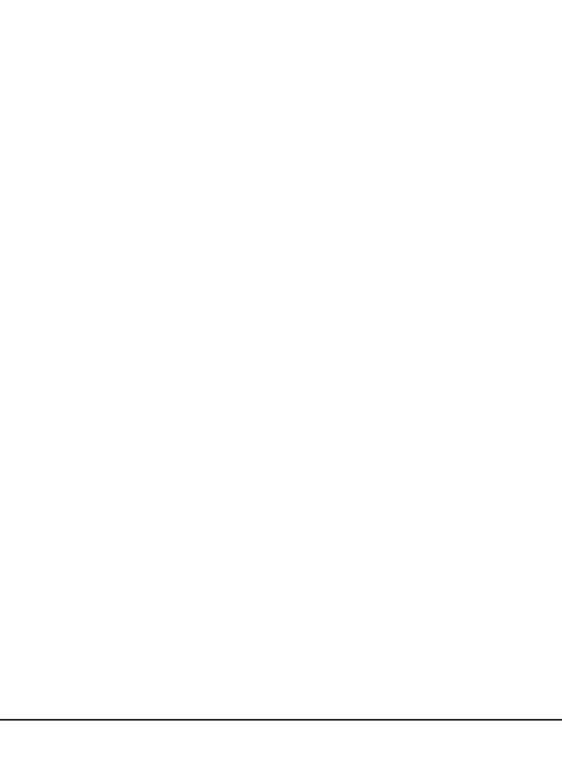

COMMENTARY

CONTINUED FROM PAGE 190

necessary, and $5 \%$ did not know they are used in dermatology. Additionally, $66 \%$ (27/41) said they had not had formal presentations on personal protective equipment (PPE) or masks, though 93\% (38/41) said they wear a surgical mask during surgery. Despite the high percentage of respondents using masks, $82 \%$ (31/38) did not know what type of mask they were wearing, and the remainder wore a variety of American Society for Testing and Materials-rated (levels 1, 2, or 3) and European Standards type II (EN14683) masks. Following the presentation, $100 \%$ of respondents said they were likely to use a smoke evacuator if made available, and 100\% reported that they had a better understanding of respirators and masks.

In summary, more than $50 \%$ of dermatology trainees in our study had not been formally educated regarding PPE despite its importance during the COVID-19 pandemic. The majority of respondents were unaware of the dangers of surgical smoke, and a small number of respondents believed that smoke evacuators were not necessary or did not know that they were even used in dermatology.
Based on the results of this quick survey during a lecture to dermatology trainees, we believe it is important to alert educators to this knowledge gap regarding PPE in the dermatology teaching curriculum. We have shown that even a short lecture format was an effective way of disseminating information about PPE and surgical safety. We believe that safety measures are more important now during a time when risk for infection with a potentially fatal virus is high. We encourage clinical educators to emphasize the importance of personal safety to trainees during residency, especially during the COVID-19 pandemic.

\section{REFERENCES}

1. Georgesen C, Lipner SR. Surgical smoke: risk assessment and mitigation strategies. J Am Acad Dermatol. 2018;79:746-755.

2. Chapman LW, Korta DZ, Lee PK, et al. Awareness of surgical smoke risks and assessment of safety practices during electrosurgery among US dermatology residents. JAMA Dermatol. 2017; 153:467.

3. Givi B, Schiff BA, Chinn SB, et al. Safety recommendations for evaluation and surgery of the head and neck during the COVID-19 pandemic [published online March 31, 2020]. JAMA Otolaryngol Neck Surg. doi:10.1001/jamaoto.2020.0780. 